\title{
Determination of Ten Corticosteroids in Illegal Cosmetic Products by a Simple, Rapid, and High-Performance LC-MS/MS Method
}

\author{
Vita Giaccone, Giuseppe Polizzotto, Andrea Macaluso, \\ Gaetano Cammilleri, and Vincenzo Ferrantelli \\ Istituto Zooprofilattico Sperimentale della Sicilia “A. Mirri”, Via Gino Marinuzzi 3, 90129 Palermo, Italy \\ Correspondence should be addressed to Vita Giaccone; vitagiaccone@gmail.com
}

Received 24 May 2016; Revised 13 September 2016; Accepted 11 October 2016; Published 14 February 2017

Academic Editor: Alberto Chisvert

Copyright (c) 2017 Vita Giaccone et al. This is an open access article distributed under the Creative Commons Attribution License, which permits unrestricted use, distribution, and reproduction in any medium, provided the original work is properly cited.

\begin{abstract}
The aim of our present work was the development of a rapid high-performance liquid chromatography method with electrospray ionization and tandem mass spectrometry detection (LC-ESI-MS/MS) for the determination of several corticosteroids in cosmetic products. Corticosteroids are suspected to be illegally added in cosmetic preparations in order to enhance the curative effect against some skin diseases. Sample preparation step consists in a single extraction with acetonitrile followed by centrifugation and filtration. The compounds were separated by reversed-phase chromatography with water and acetonitrile (both with $0.1 \%$ formic acid) gradient elution and detected by ESI-MS positive and negative ionization mode. The method was validated at the validation level of $0.1 \mathrm{mg} \mathrm{kg}^{-1}$. Linearity was studied in the $5-250 \mu \mathrm{g} \mathrm{L}^{-1}$ range and linear coefficients $\left(r^{2}\right)$ were all over 0.99 . The accuracy and precision of the method were satisfactory. The LOD ranged from 0.085 to $0.109 \mathrm{mg} \mathrm{kg}^{-1}$ and the LOQ from 0.102 to $0.121 \mathrm{mg} \mathrm{kg}^{-1}$. Mean recoveries for all the analytes were within the range 91.9-99.2\%. The developed method is sensitive and useful for detection, quantification, and confirmation of these corticosteroids in cosmetic preparations and can be applied in the analysis of the suspected samples under investigation.
\end{abstract}

\section{Introduction}

Corticosteroids are known to be highly effective drugs widely used for the treatment of inflammatory diseases. In dermatology, they were used for the treatment of skin disorders such as psoriasis, dermatoses, and eczema. They reduce inflammation and can temporarily relieve the symptoms of inflammatory skin problems of severe plaque psoriasis. For topical use they are available in the form of creams, gels, and ointments with different potency and efficacy.

Side effects and efficacy have to be related to their antiinflammatory propriety, though no active principle shows better risks/benefits ratio compared to the others [1]. The best method to evaluate the potency of corticosteroids for topical usage is the vasoconstriction test, which allows assessing the vasoconstrictor effect induced by formulations for topical usage in healthy subjects [2]. However, such test is not quite accurate because it does not consider the treatment period and frequency nor the individual response [3]. Currently topical corticosteroids are classified into seven groups according to their potency [4]. Active principles with low potency can be used for a long time and on wide skin surface; conversely, the principles with high potency should be used for short time and not applied on sensitive skin areas, such as face and armpits [5].

Prolonged therapy with corticosteroids preparations may result in adverse effects like skin atrophy, cutaneous reactivity and some systematic side effects, hypertension, diabetes mellitus, osteoporosis, allergic contact dermatitis, Cushing's syndrome, and so forth [6].

For this reason, cosmetic products should not contain glucocorticoids; nevertheless, some cosmetic preparations intended for treatment of seborrhea or psoriasis are indicated as capable of giving improvement, without clearly showing the content of glucocorticoids. Cosmetic products have no therapeutic purposes and must not claim any therapeutic 


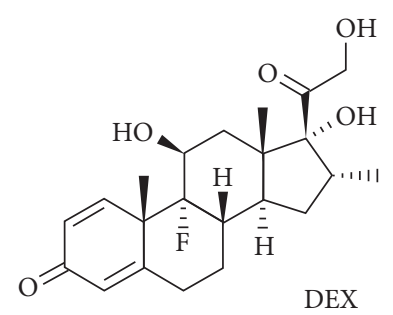<smiles>C[C@H]1[C@@H]2CCC3=CC(=O)C=C[C@]3(C)[C@H]2[C@H]2CC[C@@](O)(C(=O)CO)[C@@H]12</smiles><smiles>C[C@]12C=CC(=O)C=C1CC[C@H]1[C@@H]2CC(=O)C[C@]1(C)C(=O)CO</smiles>

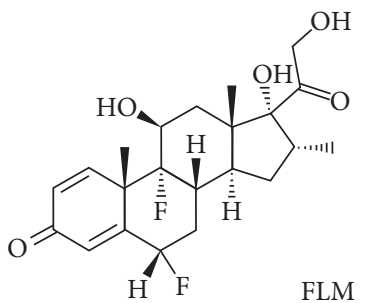

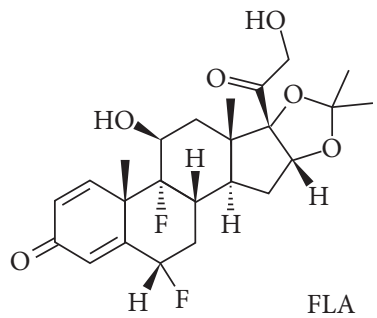<smiles>CCC(=O)O[C@]1(C(=O)CCl)[C@@H](C)C[C@H]2[C@H]3CCC4=CC(=O)C=C[C@]4(C)[C@H]3[C@@H](O)C[C@]21C</smiles>

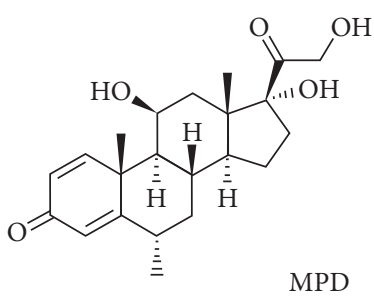

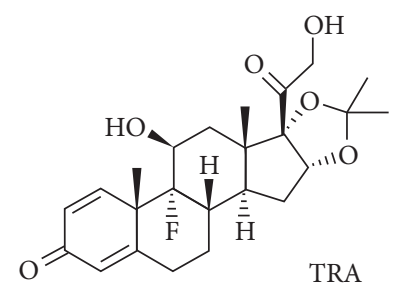

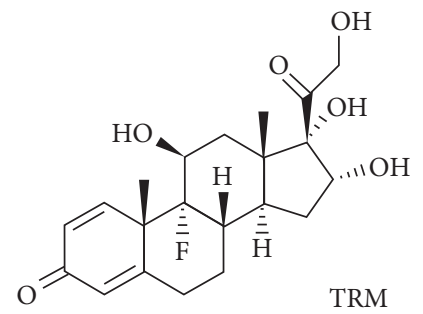<smiles>CCC(=O)OCC(=O)[C@@]1(OC(=O)CC)[C@H](C)CC2C3CCC4=CC(=O)C=C[C@]4(C)[C@@]3(Cl)[C@H](O)C[C@]21C</smiles>

FIgURE 1: Chemical structures of corticosteroids.

action [7]. For these reasons, consumers may have risk of experiencing side effects in case of a long-term use and high doses, especially without medical supervision $[8,9]$.

Therefore, there is a need for an analytical method for rapid screening of cosmetic products such as creams, ointments, and gels, which are banned in the presence of glucocorticoids and sold without health care. Earlier papers reported a number of different LC methods for these steroids in biological matrices or pharmaceutical formulations [1015]; the goal of this paper is to report the simultaneous determination of a pool of 10 different active ingredients using a simple and rapid method. In this study a simple UHPLC separation method with ESI-MS-MS detection for investigating the illegal presence of methylprednisolone (MPD), dexamethasone (DEX), prednisolone (PDL), fluocinolone acetonide (FLA), flumetasone (FLM), prednisone (PDN), triamcinolone (TRM), triamcinolone acetonide (TRA), beclomethasone (BCL), and clobetasol propionate (CLP) in cosmetic preparations was developed. We choose to investigate these specific corticosteroids because they are the most used ones in dermatologic field and to ensure that the investigation is carried out as widely as possible. In the above-mentioned classification, CLP $0.05 \%(\mathrm{v} / \mathrm{v})$ is classified in class I, with a potency that is 1800 times higher compared to hydrocortisone [16]. DEX, FLM, MPD, and PDL are classified in class VII, with TRA classified in class III. FLA is classified in class VI. Chemical structures of the target analytes are reported in Figure 1.

Although it is known that these drugs, in the case of cosmetic products, should be present at comparable concentration of the pharmaceutical formulation to induce a pharmacologic effect, we choose a very sensitive and specific method such as LC-MS/MS to identify these analytes at very low concentrations. The method was validated for linearity, accuracy, precision, and sensitivity by analyzing different pharmaceutical formulations as complex matrices.

\section{Materials and Methods}

2.1. Chemicals, Solvents, and Samples. Methanol and acetonitrile and formic acid 99.9\% (LC-MS grade) and water 
(HPLC gradient grade) were supplied from VWR (VWR International PBI Srl, Milan, Italy). Methylprednisolone, dexamethasone, prednisolone, fluocinolone acetonide, flumetasone, prednisone, triamcinolone, triamcinolone acetonide, beclomethasone, clobetasol propionate, dexamethasone $\mathrm{D} 4$, methylprednisolone D2, and prednisolone D6 (purity > 98\%) were purchased from Sigma-Aldrich (Milan, Italy). A $1000 \mathrm{mg} \mathrm{L}^{-1}$ stock solution was made by dissolving the standard in methanol. From this solution, a $10 \mathrm{mg} \mathrm{L}^{-1}$ work solution was made by dilution in methanol.

2.2. Sample Preparation. About $2 \mathrm{~g}$ of the sample was mixed to attain a homogeneous mixture; $1 \mathrm{~g}$ of the homogenized mixture was accurately weighed into a $15 \mathrm{~mL}$ centrifuge tube. The sample was spiked with $100 \mu \mathrm{L}$ of the mixed solution of internal standard (IS) at $10 \mathrm{mg} \mathrm{L}^{-1}$ and then was treated with $10 \mathrm{~mL}$ of acetonitrile, shaken by vortex for $30 \mathrm{~s}$ and by automatic shaker for 10 minutes. The solution was centrifuged for $5 \mathrm{~min}$ at $4000 \mathrm{rpm}$ and the supernatant was filtered through a $0.45 \mathrm{~mm}$ cellulose acetate filter. Finally, the solution containing the sample was transferred into a $1 \mathrm{~mL}$ vial.

2.3. Chromatographic Conditions. LC analysis was carried out through a Thermo Fischer UHPLC system (Thermo Fisher Scientific, California, USA) constituted of an ACCELA 1250 quaternary pump equipped with a degasser, a ACCELA autosampler equipped with column oven, and a Rheodyne valve with $25 \mu \mathrm{L}$ sample loop. Chromatographic separation was obtained using a Thermo Scientific Hypersil Gold PFP reversed-phase UHPLC column $(100 \mathrm{~mm}, 2.1 \mathrm{~mm}$ ID, and $1.9 \mu \mathrm{m})$. The LC eluents were water (A) and acetonitrile (B), everyone containing $0.1 \%(\mathrm{v} / \mathrm{v})$ formic acid. The gradient was initiated with $70 \%$ eluent $\mathrm{A}$ and $30 \%$ eluent $\mathrm{B}$ for $0.5 \mathrm{~min}$, continued with linear variation to $20 \% \mathrm{~A}$ and $80 \% \mathrm{~B}$ in $6.5 \mathrm{~min}$; this condition was maintained for $0.5 \mathrm{~min}$. The system returned to $70 \% \mathrm{~A}$ and $30 \% \mathrm{~B}$ in $0.5 \mathrm{~min}$ and was reequilibrated for $2 \mathrm{~min}$. The column temperature was $30^{\circ} \mathrm{C}$ and the sample temperature was kept at $6^{\circ} \mathrm{C}$. The flow rate was $0.4 \mathrm{~mL} \mathrm{~min}^{-1}$ and the injection volume was $5 \mu \mathrm{L}$.

2.4. MS Conditions. The mass spectrometer was a triple quadrupole TSQ Vantage (Thermo Fisher Scientific, California, USA) in positive and negative electrospray ionization mode (ESI). Product ion scans of each analyte were performed by direct infusion $\left(10 \mu \mathrm{L} \mathrm{min}^{-1}\right)$ of $1 \mathrm{mg} \mathrm{L}^{-1}$ individual standard solutions with the built-in syringe pump through a T-junction, mixing with the blank column eluate $\left(200 \mu \mathrm{L} \mathrm{min}^{-1}\right)$.

ESI parameters optimized were as follows: capillary voltage $4.5 \mathrm{kV}$; capillary temperature $310^{\circ} \mathrm{C}$; vaporizer temperature $150^{\circ} \mathrm{C}$; sheath and auxiliary gas pressure were fixed at 35 and 10 (arbitrary units), respectively. The collision gas was argon at 1.5 mTorr and peak resolution of $0.7 \mathrm{FWHM}$ was used on Q1 and Q3. The scan time for each monitored transition was $0.01 \mathrm{~s}$ and the scan width was $0.01 \mathrm{~m} / z$. The collision energy parameters associated with the precursor and the product ions are given in Table 1. Acquisition data were recorded and elaborated using Xcalibur ${ }^{\mathrm{TM}}$ version 2.1.0.1139 software from Thermo.

2.5. Chromatography and Quantitative Determination. The presence of corticosteroids was verified by comparison between the chromatograms of the standard solution and the sample: the retention time and the relative abundances of the fragment were compared. For the quantitative determination, we used two methods: interpolation of the signal from the analytes of the sample in the solution calibration curve and the standard addition method. The calibration curve for the standard solutions was made with the concentration levels of 5-25-50-100-150-250 $\mu \mathrm{g} \mathrm{L}^{-1}$. For the standard addition method, we chose a cream containing a known amount of clobetasol propionate declared by the pharmaceutical company $(0.05 \%, \mathrm{w} / \mathrm{w})$. We made three spiked levels of concentration in order to have twice, three times, and five times the declared content of drug in the cream sample. The samples, thus obtained, were diluted, extracted, and analyzed. Then we calculated the concentration of the analyte by the extrapolation of the value through the intersection of the calibration curve and the $x$-axis. Areas used for the quantification are generated by the base peak signal only.

2.6. Validation Procedure. For the estimation of the validation parameters, blank samples were fortified at three different concentrations in equidistant steps: $0.5,1.0$, and $1.5 \mathrm{mg} \mathrm{kg}^{-1}$. Six spiked samples, at each of the three levels, were analyzed. The 18-replicate analyses (six for each level) were repeated in three separate days giving 54 independent determinations.

Linearity, specificity, recovery, matrix effect, limit of detection (LOD), limit of quantification (LOQ), precision (repeatability and the within-laboratory reproducibility), and accuracy were measured.

To test the selectivity/specificity of the method, 20 blank samples of different type (creams, gels, and ointments taken from make-up shops) were analyzed to verify the absence of potential interfering compounds at analytes retention time. Linearity was studied in the range of 6-point calibration curve for all the analytes. The recoveries were obtained using six replicates at each level. For the evaluation of matrix effects, three preparations were compared: the first is a blank sample spiked at $1.0 \mathrm{mg} \mathrm{kg}^{-1}$ and analyzed after the extraction procedure. The second is a blank matrix extract spiked immediately before LC injection. The third is a mix of the target analytes corresponding to spiked level.

Precision is expressed as the percent relative standard deviation (RSD\%) of concentrations calculated for spiked samples and accuracy as the relative error of the calculated concentrations. Both parameters were measured in intra- and interday manner. The accuracy was tested also analyzing a pharmaceutical preparation containing a declared concentration of clobetasol propionate and comparing the results to the true concentration.

The limit of detection (LOD) was estimated on the basis of the results for six replicates of cream sample spiked at the $0.1 \mathrm{mg} \mathrm{kg}^{-1}$ level and was calculated using the formula: 
TABLE 1: Parameters for SRM acquisition of the corticosteroids. ${ }^{a}$ The most abundant product ion.

\begin{tabular}{|c|c|c|c|c|c|c|}
\hline Analyte & Internal standard & Retention time (min) & ESI & $\begin{array}{c}\text { Precursor ion }(m / z) \\
{[\mathrm{M}+\mathrm{HCOO}]^{-} \text {or }[\mathrm{M}+\mathrm{H}]^{+}}\end{array}$ & Product ions $(m / z)$ & Collision energy $(\mathrm{eV})$ \\
\hline \multirow{2}{*}{ MPD } & \multirow{2}{*}{ MPD D2 } & \multirow{2}{*}{2.16} & neg & \multirow{2}{*}{419.1} & $343.0^{\mathrm{a}}$ & 20 \\
\hline & & & neg & & 308.9 & 32 \\
\hline \multirow{2}{*}{ DEX } & \multirow{2}{*}{ DEX D4 } & \multirow{2}{*}{2.35} & neg & \multirow{2}{*}{437.1} & $361.0^{\mathrm{a}}$ & 19 \\
\hline & & & neg & & 307.0 & 30 \\
\hline \multirow{2}{*}{ PDL } & \multirow{2}{*}{ PDL D6 } & \multirow{2}{*}{1.61} & neg & \multirow{2}{*}{405.1} & $328.9^{\mathrm{a}}$ & 19 \\
\hline & & & neg & & 294.9 & 32 \\
\hline \multirow{2}{*}{ FLA } & \multirow{2}{*}{-} & \multirow{2}{*}{3.30} & neg & \multirow{2}{*}{497.1} & $431.0^{\mathrm{a}}$ & 21 \\
\hline & & & neg & & 354.8 & 22 \\
\hline \multirow{2}{*}{ FLM } & \multirow{2}{*}{-} & \multirow{2}{*}{2.69} & neg & \multirow{2}{*}{455.1} & $379.0^{\mathrm{a}}$ & 19 \\
\hline & & & neg & & 324.8 & 30 \\
\hline \multirow{2}{*}{ PDN } & \multirow{2}{*}{-} & \multirow{2}{*}{1.82} & neg & \multirow{2}{*}{403.1} & $327.1^{\mathrm{a}}$ & 17 \\
\hline & & & neg & & 299.1 & 20 \\
\hline \multirow{2}{*}{ TRM } & \multirow{2}{*}{-} & \multirow{2}{*}{1.24} & neg & \multirow{2}{*}{439.1} & $344.9^{\mathrm{a}}$ & 23 \\
\hline & & & neg & & 325.0 & 24 \\
\hline \multirow{2}{*}{ TRA } & \multirow{2}{*}{-} & \multirow{2}{*}{2.95} & neg & \multirow{2}{*}{479.1} & $374.9^{\mathrm{a}}$ & 25 \\
\hline & & & neg & & 336.8 & 19 \\
\hline \multirow{2}{*}{ BCL } & \multirow{2}{*}{-} & 262 & neg & 4530 & $407.0^{\mathrm{a}}$ & 14 \\
\hline & & 2.02 & neg & 453.0 & 376.9 & 16 \\
\hline CLP & - & 5.14 & pos & 4672 & $447.2^{\mathrm{a}}$ & 10 \\
\hline CLr & - & 0.14 & pos & $40 \% .2$ & 373.0 & 12 \\
\hline DEX D4 & & 2.38 & neg & 441.1 & 363.2 & 20 \\
\hline MPD D2 & & 2.17 & neg & 421,1 & 342.8 & 19 \\
\hline PDL D6 & & 1.61 & neg & 411.1 & 333.0 & 18 \\
\hline
\end{tabular}

TABLE 2: Validation data of linearity and LOD and LOQ for samples containing spiked standard solutions in blank cosmetic preparations. ${ }^{\mathrm{a}}$ Internal calibration. ${ }^{\mathrm{b}}$ Concentration in $\mathrm{mg} \mathrm{kg}^{-1}$.

\begin{tabular}{lcccc}
\hline \multirow{2}{*}{ Steroids } & \multicolumn{4}{c}{ Standard solutions } \\
& Slope & $r^{2}$ & LOD $^{\mathrm{b}}$ & LOQ $^{\mathrm{b}}$ \\
\hline CLP $^{\mathrm{a}}$ & 0.008 & 0.999 & 0.089 & 0.102 \\
DEX $^{\mathrm{a}}$ & 0.058 & 0.999 & 0.109 & 0.117 \\
MPD $^{\mathrm{a}}$ & 0.062 & 0.998 & 0.103 & 0.121 \\
PDL $^{\mathrm{a}}$ & 0.055 & 0.999 & 0.085 & 0.099 \\
FLA & 1553 & 0.996 & 0.093 & 0.107 \\
FLM & 13892 & 0.998 & 0.097 & 0.105 \\
PDN & 4123 & 0.999 & 0.098 & 0.112 \\
TRM & 1741 & 0.998 & 0.092 & 0.101 \\
TRA & 1635 & 0.997 & 0.101 & 0.113 \\
BCL & 1986 & 0.998 & 0.104 & 0.117 \\
\hline
\end{tabular}

LOD $=\bar{X}+3$ SD, where $\bar{X}$ is the mean of the calculated concentration and $\mathrm{SD}$ is the standard deviation of replicate analyses. The quantification limit (LOQ) was calculated using the formula: $\mathrm{LOQ}=\bar{X}+10 \mathrm{SD}$.

The calculated LOD and LOQ values are reported in Table 2.

\section{Results and Discussion}

3.1. Chromatography and Validation Results. The tunes of the MS conditions for standards and the deuterated ISs were performed by direct infusion of $1 \mathrm{mg} \mathrm{L}^{-1}$ individual standard solutions with the built-in syringe pump. It was found that the precursor ions with the most abundant signal are composed of the formate adduct, $[\mathrm{M}+\mathrm{HCOO}]^{-}$, in electrospray negative mode; only for CLP was the most abundant signal obtained in positive mode monitoring the adduct $[\mathrm{M}+\mathrm{H}]^{+}$. After that, we optimized the chromatographic conditions by several injections of a mixed solution of the target analytes at the concentration of $100 \mu \mathrm{g} \mathrm{L}^{-1}$ in order to test different combinations of mobile phases. Then we found the best gradient condition, reported in the experimental section of this paper, for the best symmetry and resolution of the peaks. The spectrometric determination was performed in MRM mode in order to obtain better selectivity and sensitivity.

Method validation was performed by evaluating the following parameters: linearity, limit of detection (LOD), limit of quantification (LOQ), intraday variability (repeatability), interday variability (intermediate precision), and recovery (trueness). We chose as complex matrices different cosmetic products free of analytes, such as creams and ointments taken from make-up shops.

The presence of the target substances in cosmetic samples was validated by comparing the retention time of the peak areas to a high purity standard. Also the relative abundances of the mass transitions were used as identification parameter (Figures 2-11). 

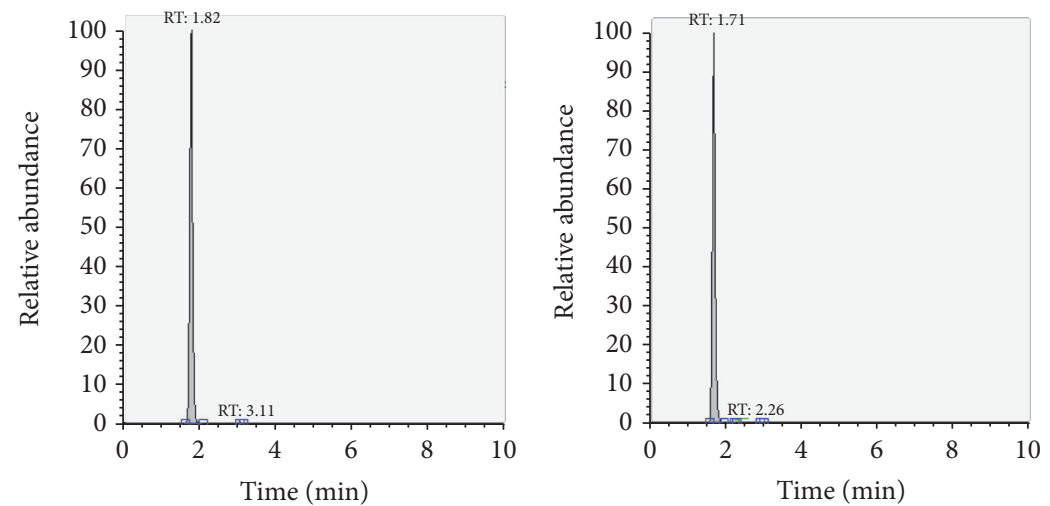

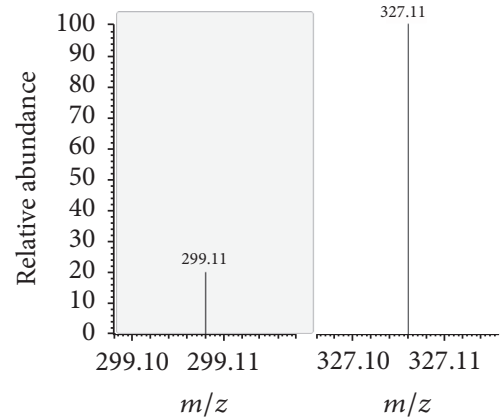

(a)

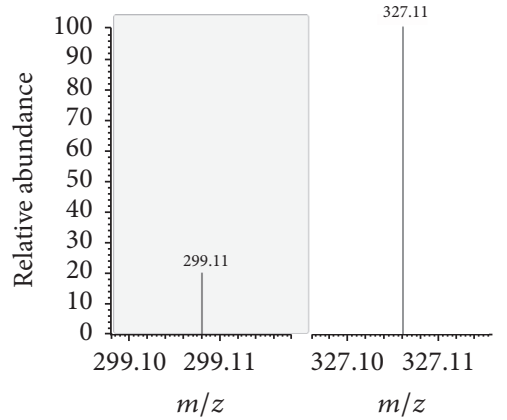

(b)

FIGURE 2: LC-MS/MS chromatograms of PDN and SRM transitions and relative abundances. (a) Standard in a $100 \mu \mathrm{g} \mathrm{L}^{-1}$ solution. (b) Standard in the spiked sample at $100 \mathrm{mg} \mathrm{kg}^{-1}$.
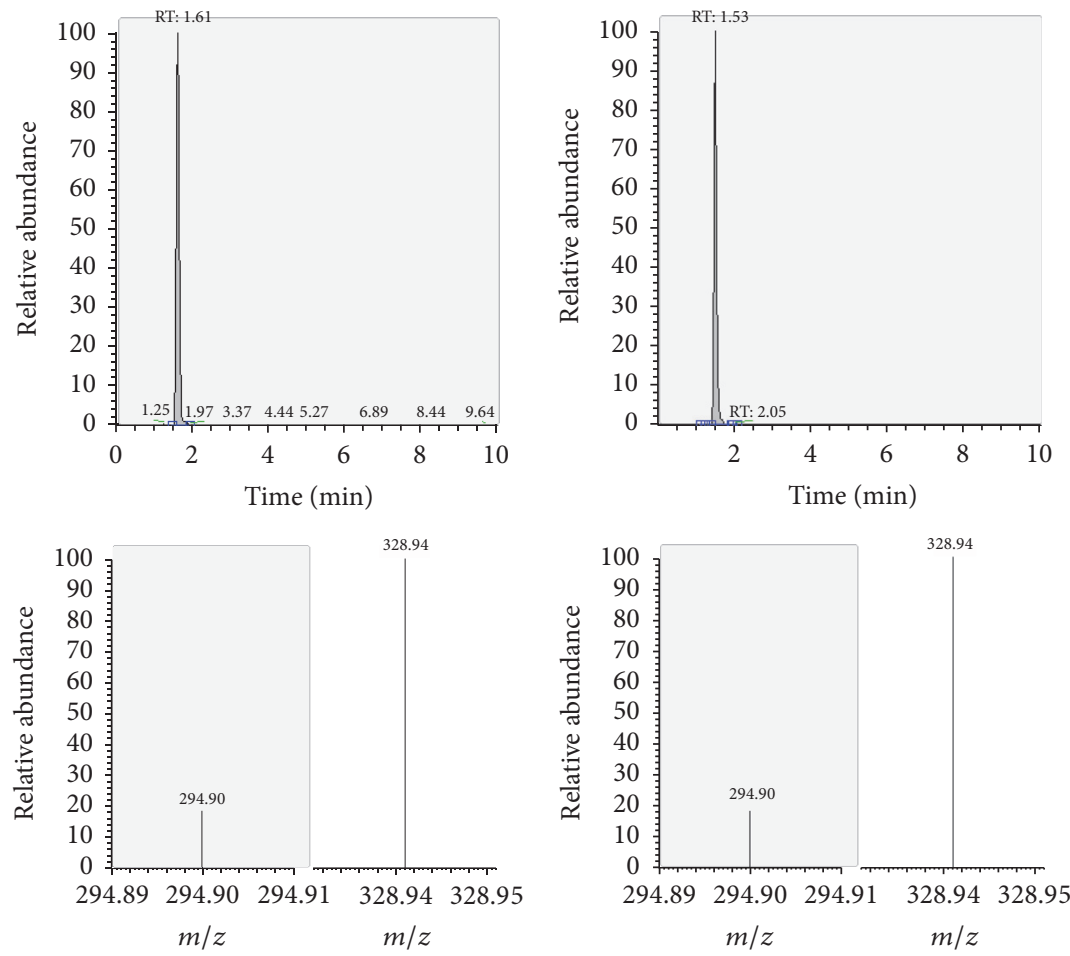

(a)

(b)

FIGURE 3: LC-MS/MS chromatograms of PDL and SRM transitions and relative abundances. (a) Standard in a $100 \mu \mathrm{g} \mathrm{L} \mathrm{L}^{-1}$ solution. (b) Standard in the spiked sample at $100 \mathrm{mg} \mathrm{kg}^{-1}$. 

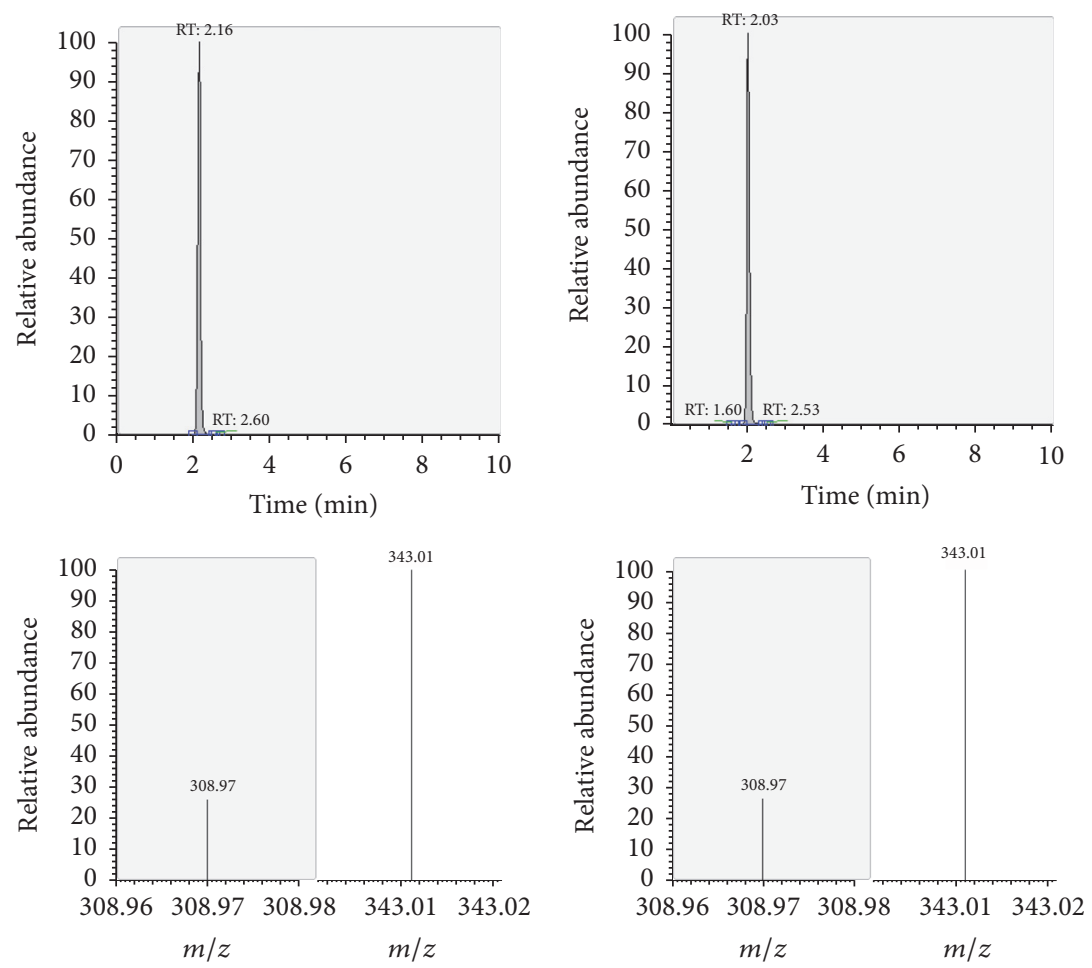

(a)

(b)

FIGURE 4: LC-MS/MS chromatograms of MPD and SRM transitions and relative abundances. (a) Standard in a $100 \mu \mathrm{g} \mathrm{L} \mathrm{L}^{-1}$ solution. (b) Standard in the spiked sample at $100 \mathrm{mg} \mathrm{kg}^{-1}$.
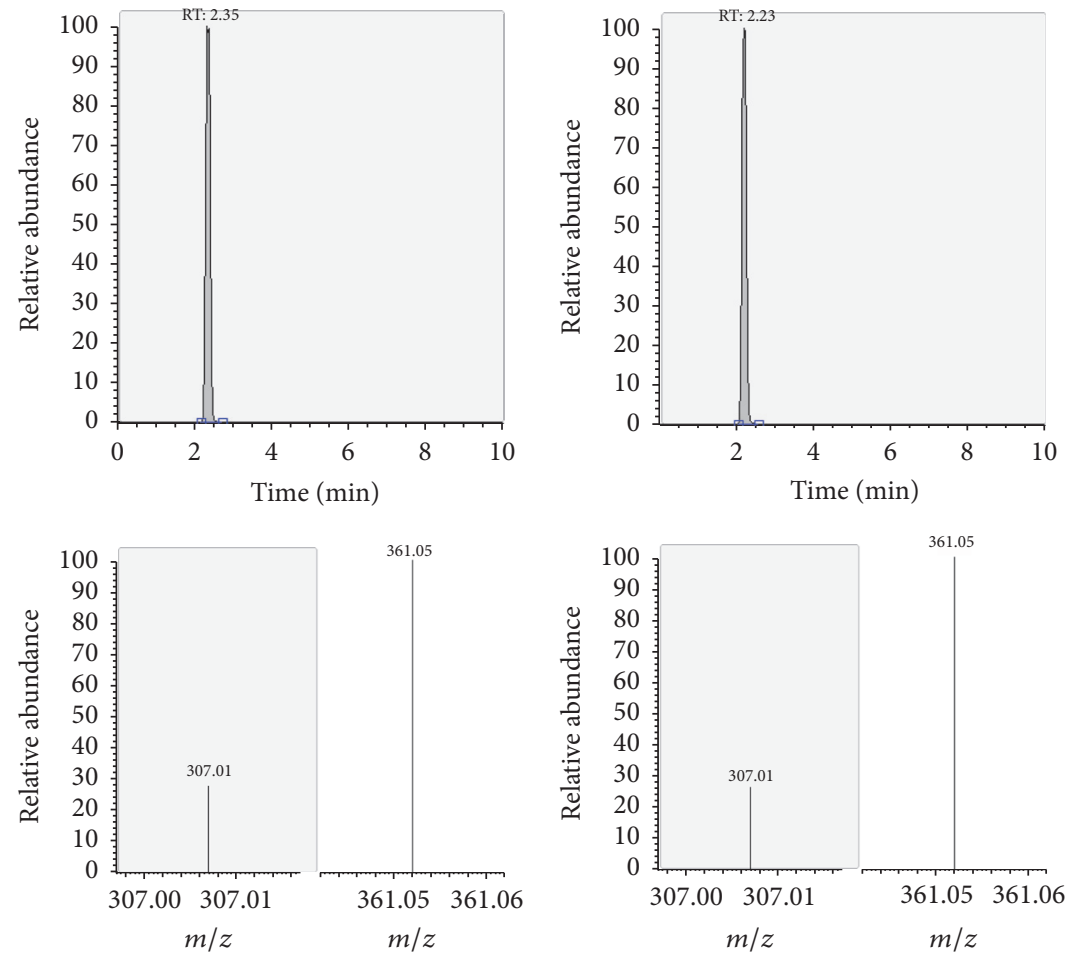

(a)

(b)

FIGURE 5: LC-MS/MS chromatograms of DEX and SRM transitions and relative abundances. (a) Standard in a $100 \mu \mathrm{g} \mathrm{L} \mathrm{L}^{-1}$ solution. (b) Standard in the spiked sample at $100 \mathrm{mg} \mathrm{kg}^{-1}$. 

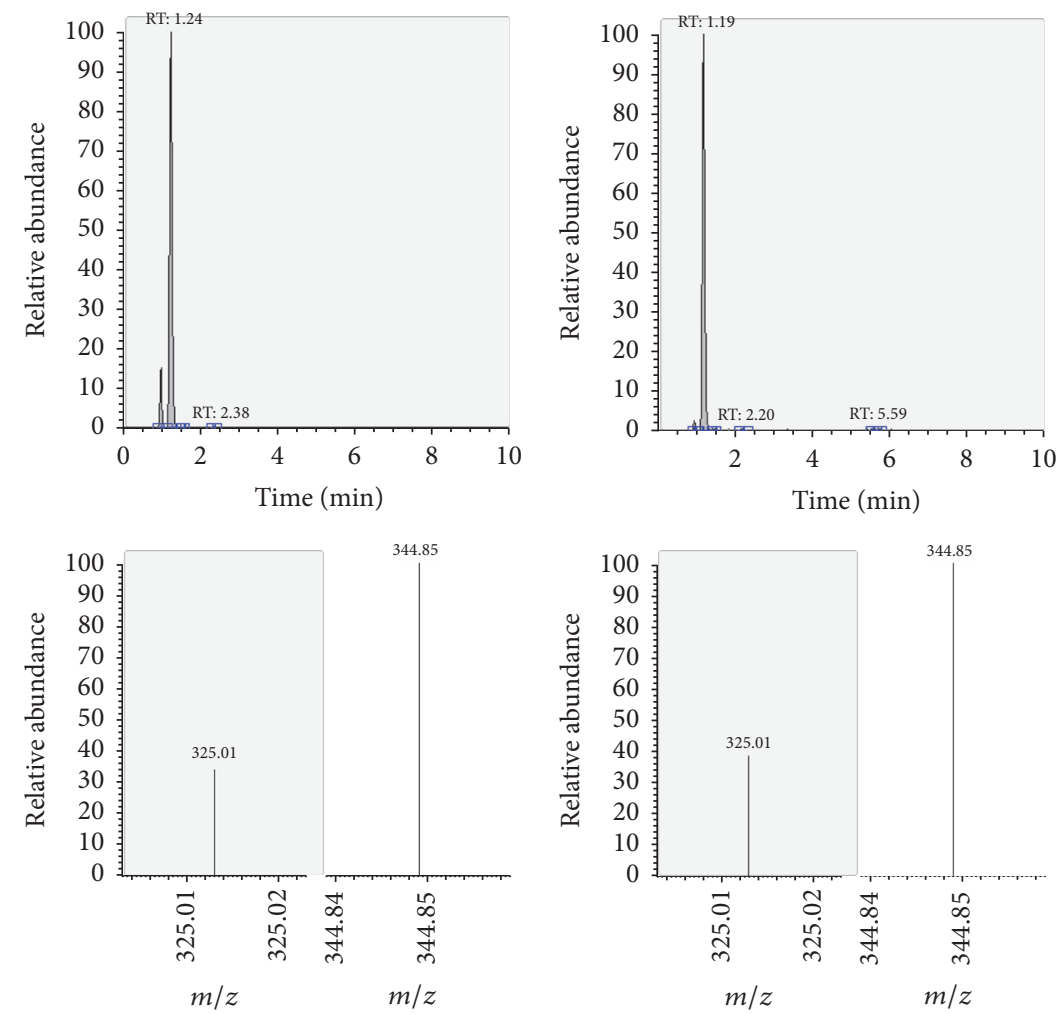

(a)

(b)

FIGURE 6: LC-MS/MS chromatograms of TRM and SRM transitions and relative abundances. (a) Standard in a $100 \mu \mathrm{g} \mathrm{L} \mathrm{L}^{-1}$ solution. (b) Standard in the spiked sample at $100 \mathrm{mg} \mathrm{kg}^{-1}$.
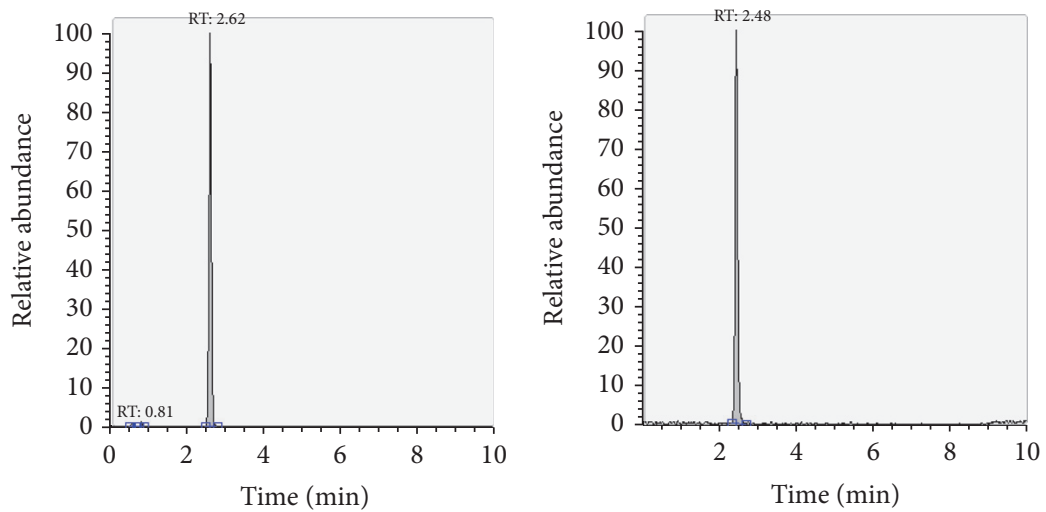

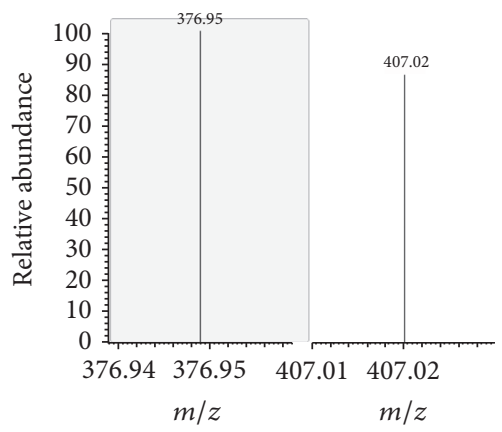

(a)

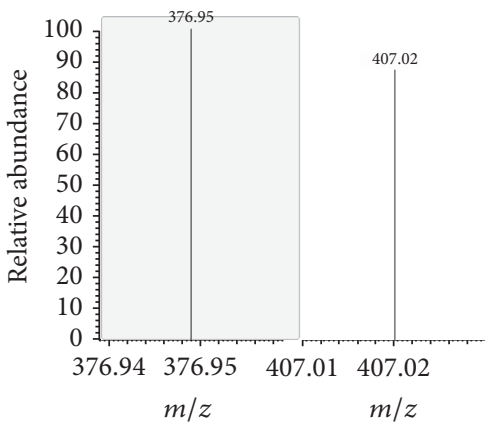

(b)

FIGURE 7: LC-MS/MS chromatograms of BCL and SRM transitions and relative abundances. (a) Standard in a $100 \mu \mathrm{g} \mathrm{L} \mathrm{L}^{-1}$ solution. (b) Standard in the spiked sample at $100 \mathrm{mg} \mathrm{kg}^{-1}$. 

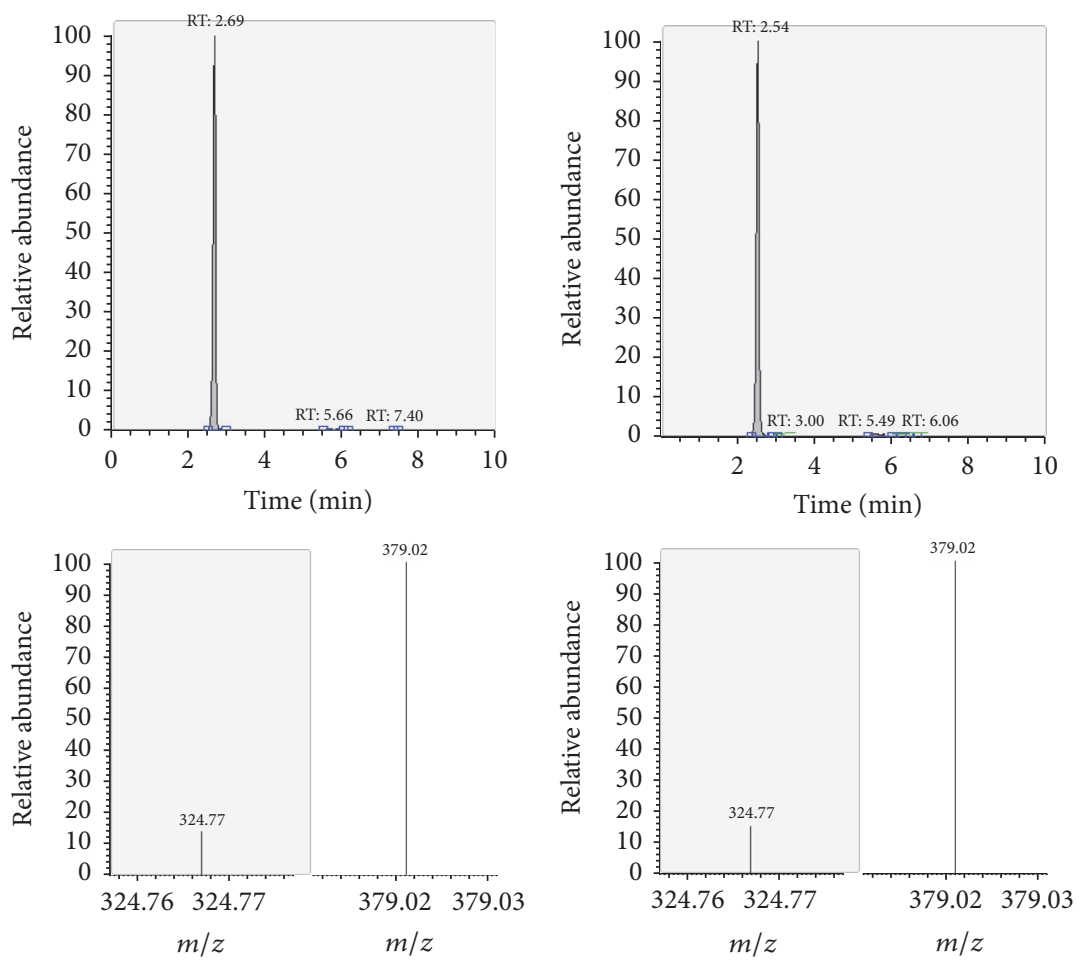

(a)

(b)

FIGURE 8: LC-MS/MS chromatograms of FLM and SRM transitions and relative abundances. (a) Standard in a $100 \mu \mathrm{g} \mathrm{L}^{-1}$ solution. (b) Standard in the spiked sample at $100 \mathrm{mg} \mathrm{kg}^{-1}$.
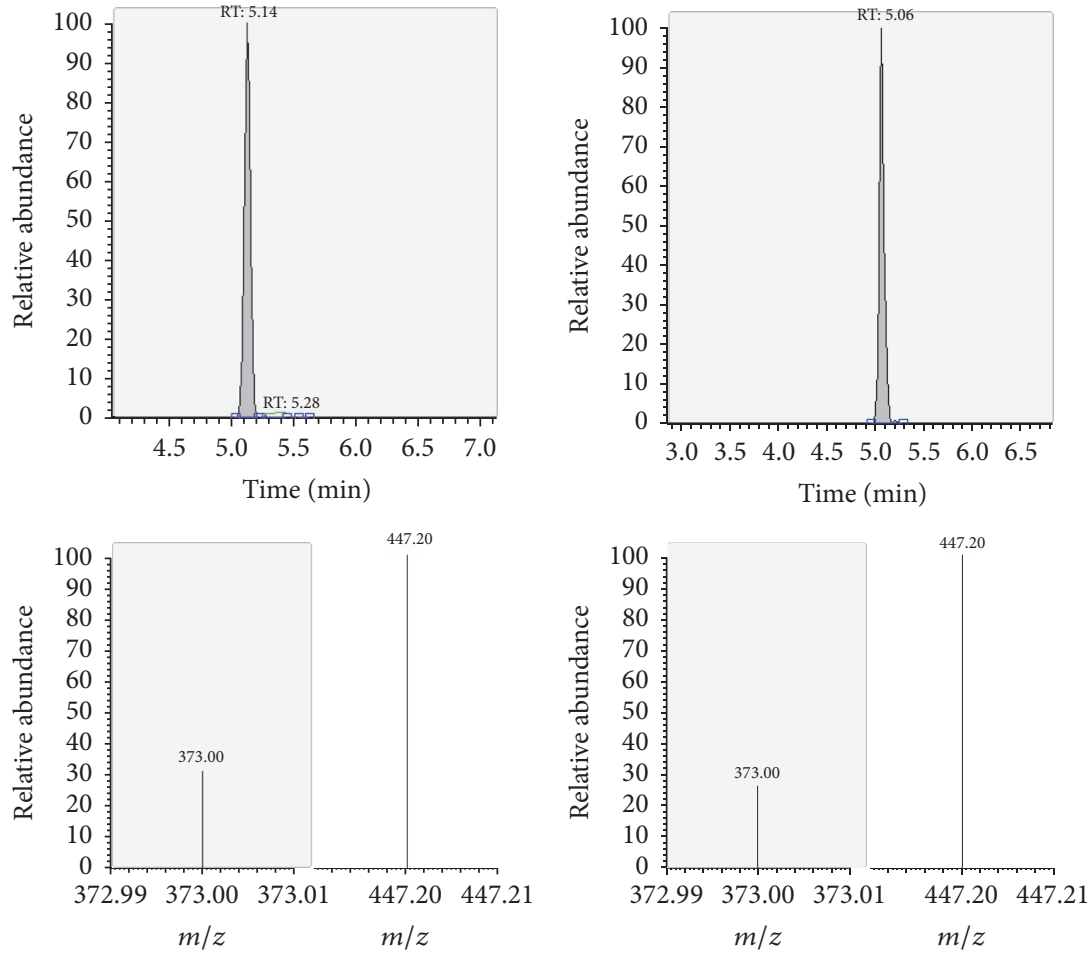

(a)

(b)

FIGURE 9: LC-MS/MS chromatograms of CLP and SRM transitions and relative abundances. (a) Standard in a $100 \mu \mathrm{g} \mathrm{L} \mathrm{L}^{-1}$ solution. (b) Standard in the spiked sample at $100 \mathrm{mg} \mathrm{kg}^{-1}$. 

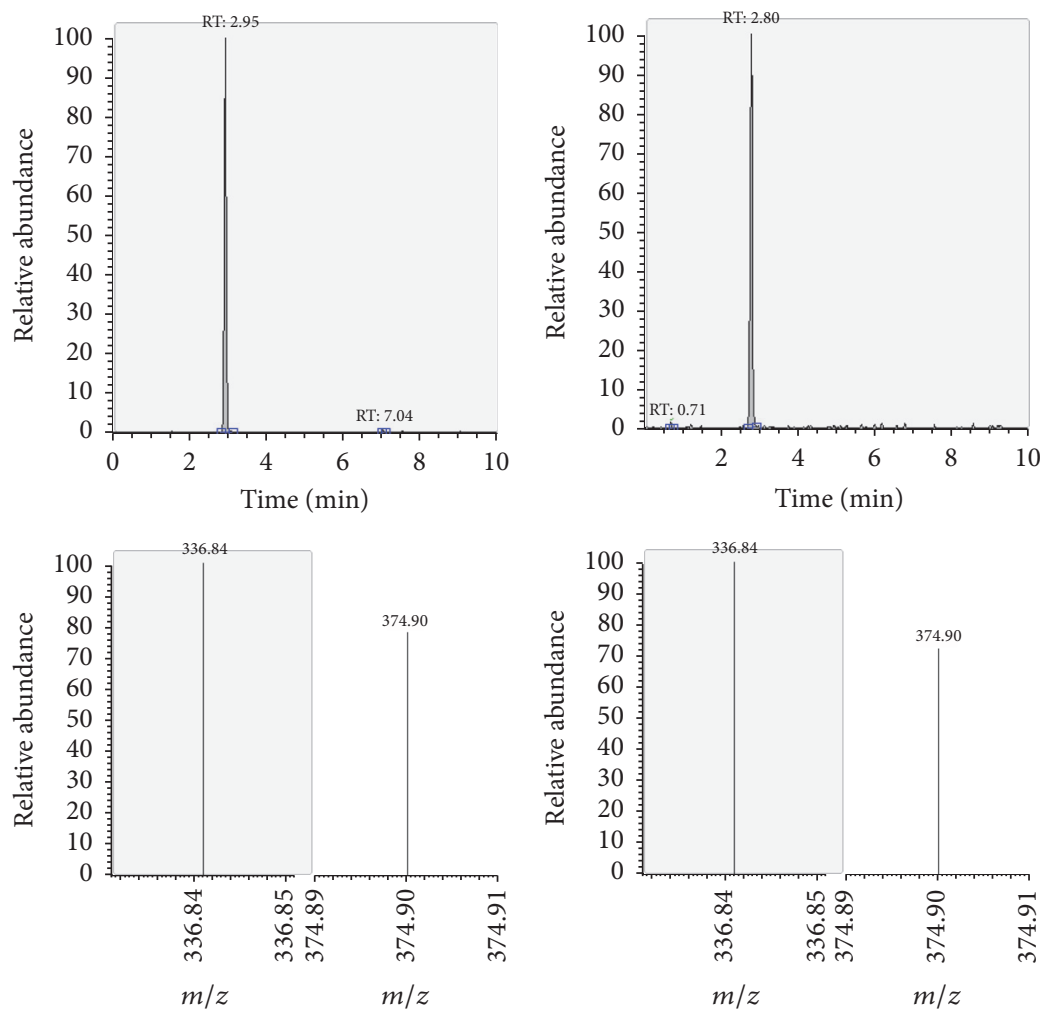

(a)

(b)

FIGURE 10: LC-MS/MS chromatograms of TRA and SRM transitions and relative abundances. (a) Standard in a $100 \mu \mathrm{g} \mathrm{L}^{-1}$ solution. (b) Standard in the spiked sample at $100 \mathrm{mg} \mathrm{kg}^{-1}$.
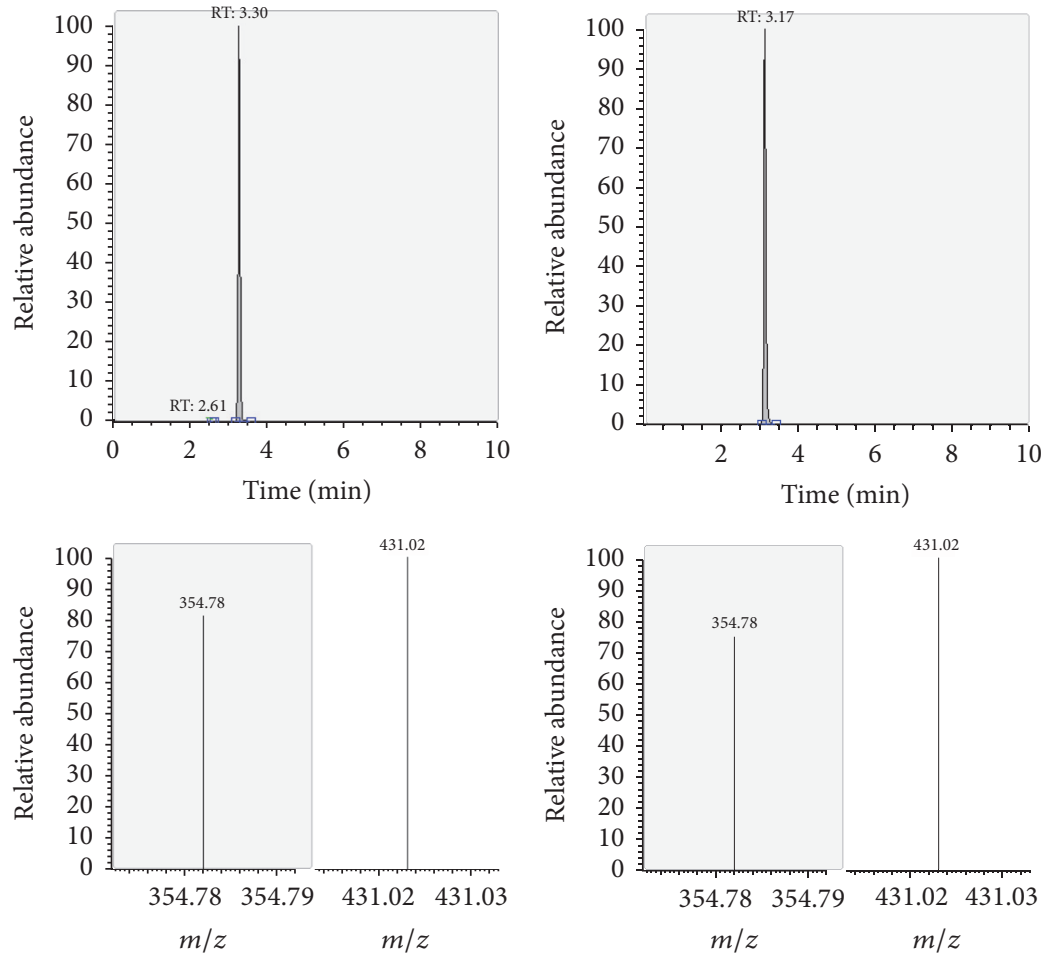

(a)

(b)

FIGURE 11: LC-MS/MS chromatograms of FLA and SRM transitions and relative abundances. (a) Standard in a $100 \mu \mathrm{g} \mathrm{L^{-1 }}$ solution. (b) Standard in the spiked sample at $100 \mathrm{mg} \mathrm{kg}^{-1}$. 

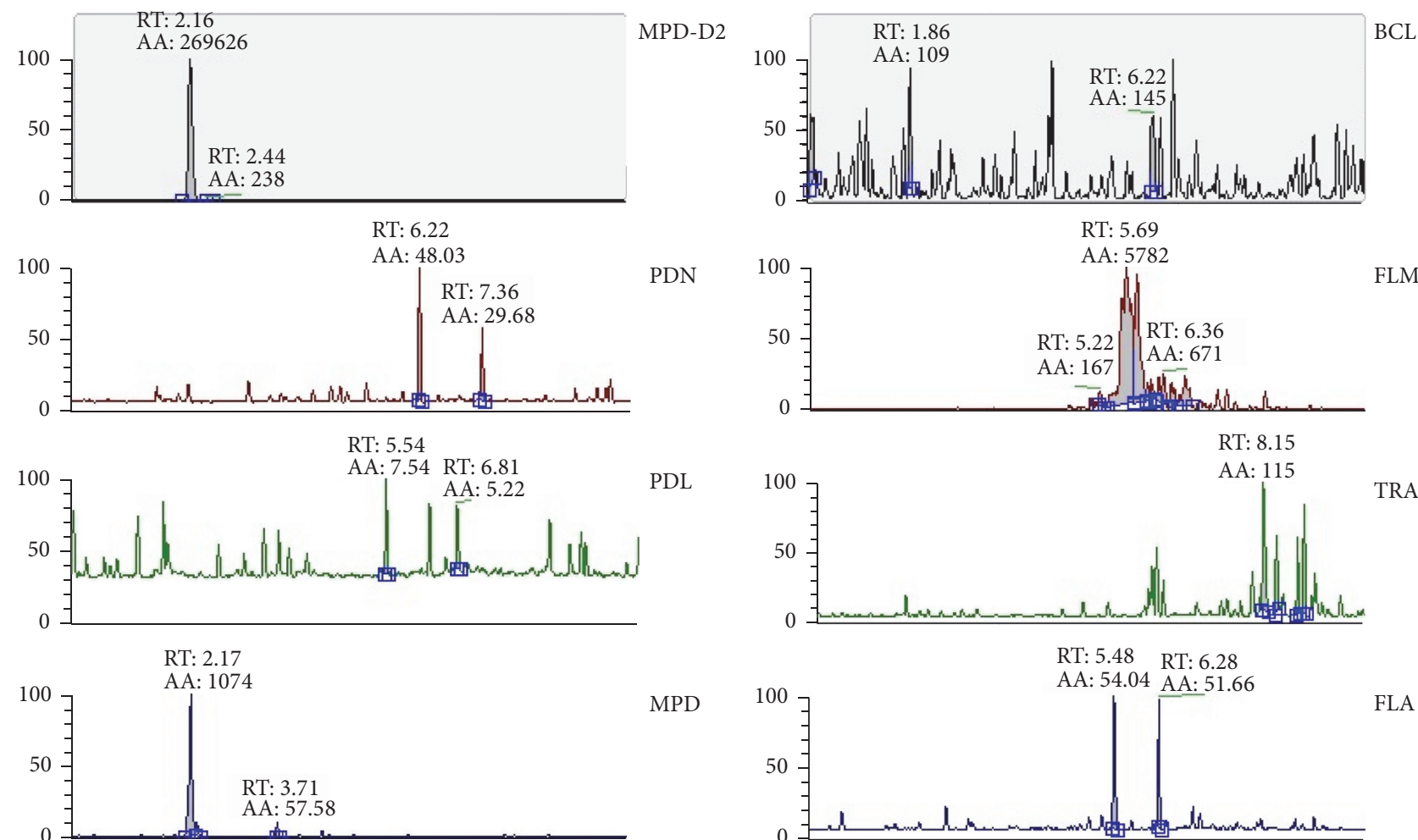

PDL

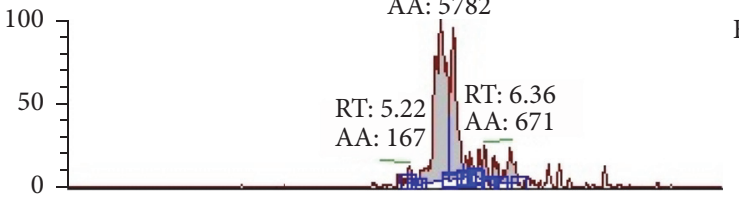

FLM
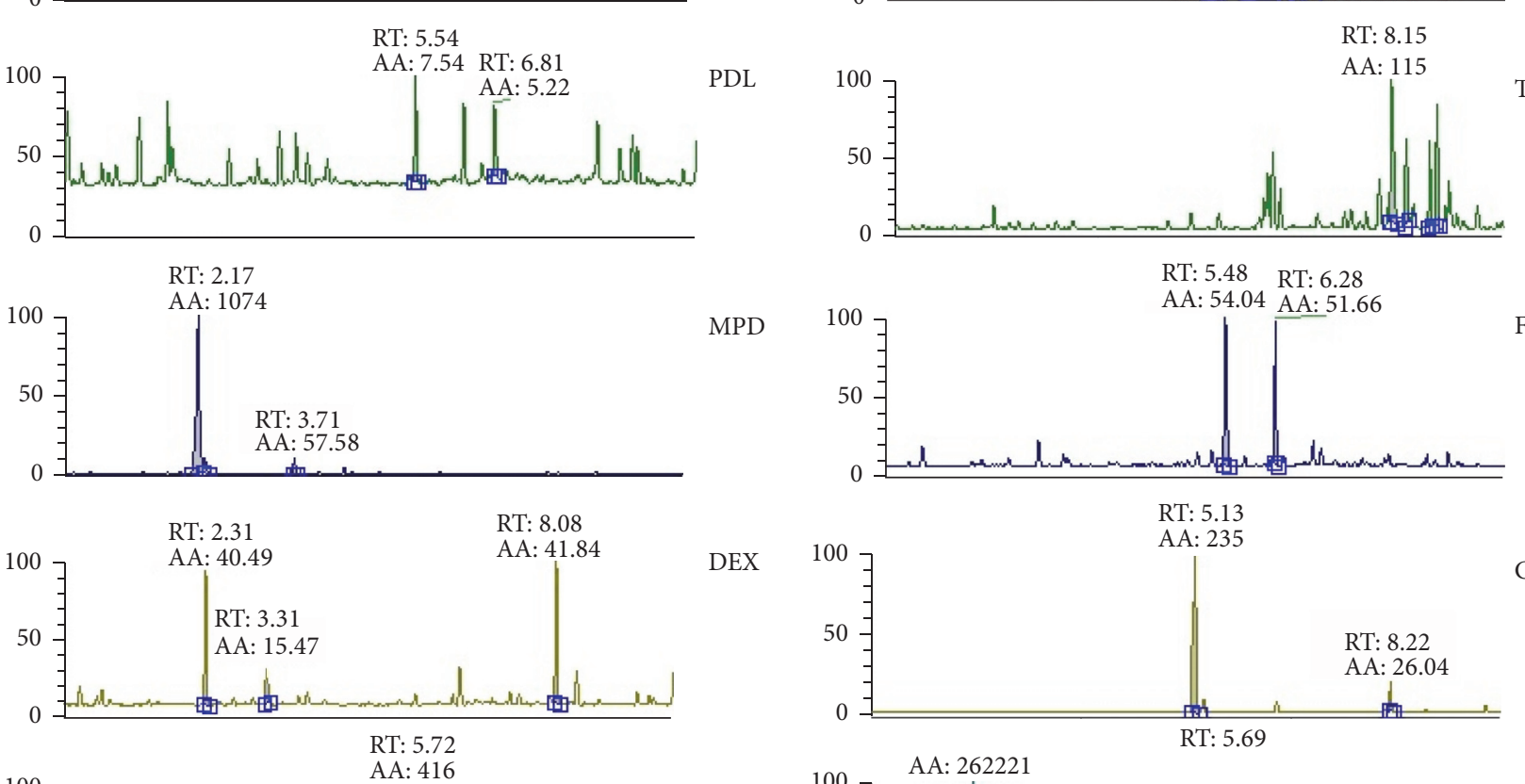

MPD
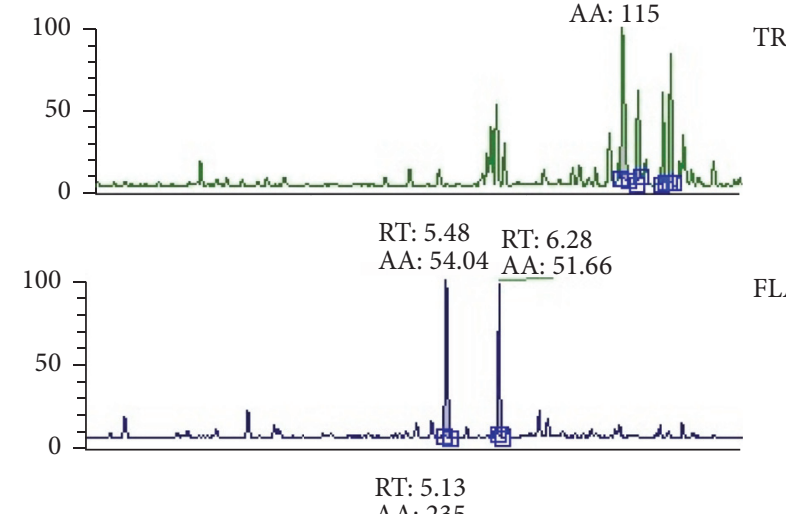

DEX
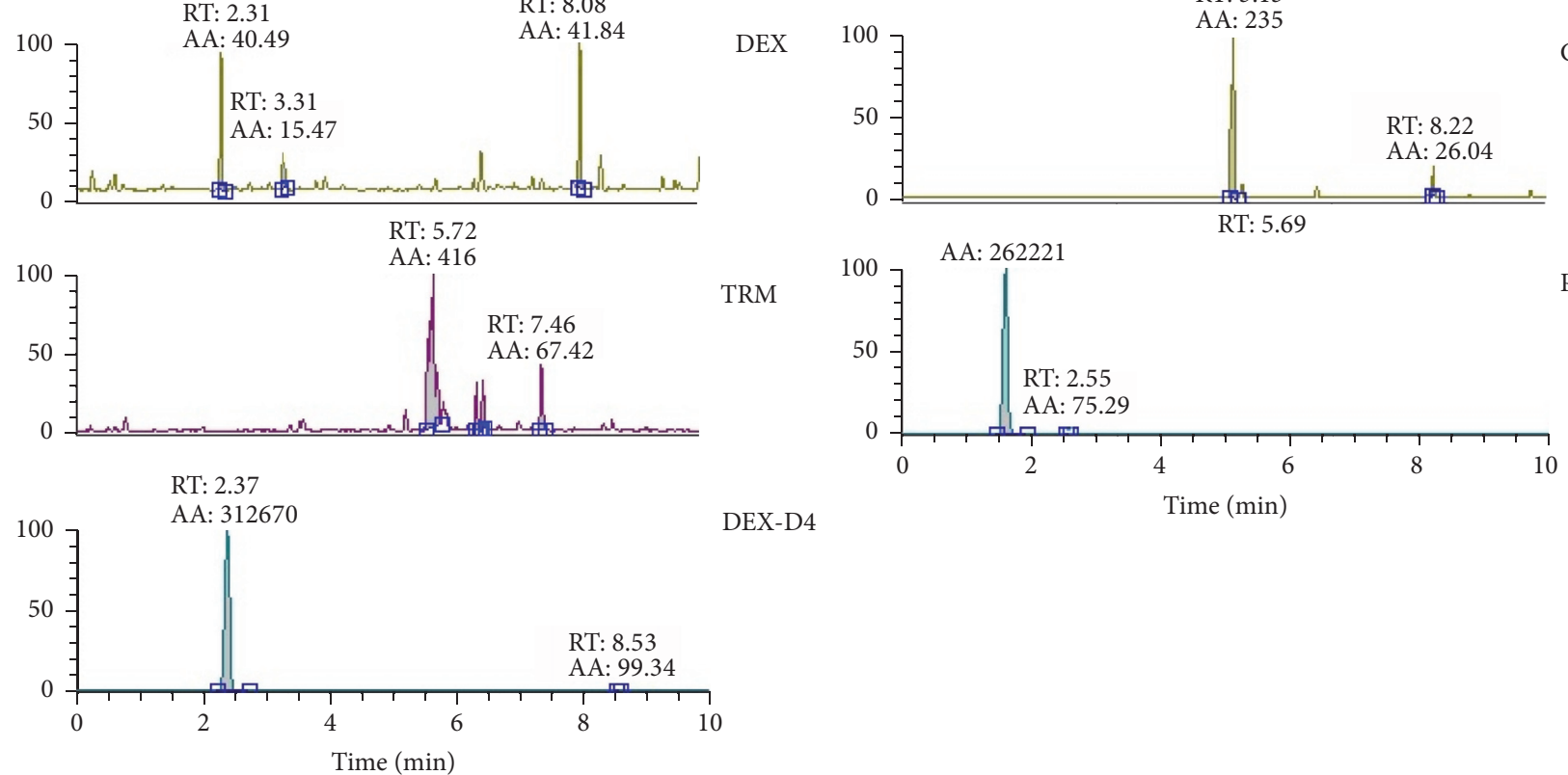

DEX-D4

FIGURE 12: Chromatograms of the corticosteroids (PDN, PDL, MPD, TRM, BCL, FLM, TRA, FLA, DEX, and CLP) and internal standards (MPD-D2, DEX-D4, and PDL-D6) in the blank sample. The relative abundance ( $y$-axis) is reported in percentage (\%). AA: peak area; RT: retention time.

Specificity was demonstrated by identifying the analytes based on the precursor and product ions as well as the relative retention times (compared to the standards). Ion ratios in matrix-matched calibrators and analytes solutions typically matched each other to around $90 \%$; hence, a maximum difference of $10 \%$ is tolerable. No interfering peaks at the RT of the analytes were found during selectivity test, consisting in a comparison between chromatogram of samples with standards in matrix and chromatogram of blank samples (Figure 12). 
Linearity was studied within a concentration range of 5$250 \mu \mathrm{g} \mathrm{L}{ }^{-1}$ for all the steroids. The linearity ranges were fixed to secure the lower range limit [17].

Analytical method guarantees safety and efficiency because it highlights any corticosteroid residue. Any samples with levels above the linearity range can be appropriately diluted.

Calibration graphs were obtained including zero plotting the ratio analyte area/internal standard area $(=y)$ versus analyte concentration $(=x)$ for the analytes with the same deuterated standards and plotting the peak areas of analyte versus the corresponding concentration $\left(\mu \mathrm{g} \mathrm{L}^{-1}\right.$ in the final dilution) for the others. A regression model was then applied to the calibration data set and linear calibration curves showed correlation coefficients $r^{2}$ higher than 0.996 . The high correlation coefficient $r^{2}$ values indicated good correlations between corticosteroids concentrations and peak areas.

Standard addition method was also applied simultaneously to confirm the linearity and to determine drugs content. In the latter method, the concentration of steroids was determined by extrapolating the $x$-intercept value from the standard addition curve. This method was applied only to CLP, obtaining comparable results with the curve calibration method (within the range of 5\%). The matrix effect was investigated in order to reveal possible ionization suppression or enhancement caused by matrix components. It was evaluated on different cosmetic products.

Two aliquots of each sample were extracted as previously described and the extracts were spiked of analytes and IS. At the same time, a solution of the detected analytes was prepared at the same concentration level. No significant ionic enhancement was found for each analyte and the absolute analytical recoveries obtained for spiked samples of three different concentrations were around 90\%, excluding signal suppression or interferences due to endogenous substances in the complex matrix. LOD and LOQ values were found to be suitable for the purposes of the present study (Table 2). Particularly, the calculated LOQ tested for precision and accuracy presented RSD always lower than 20\%. Excellent results were obtained for precision and accuracy of intraday and interday analyses with relative standard deviation (RSD) values within $10 \%$, responding to established acceptance criteria $[18,19]$. The internal standards were used as surrogate to measure the overall efficiency of the method (recovery) during its routine use. Trueness was expressed in terms of recovery rates; the values were in the range of $91-99 \%$. Validation data are listed in Tables 2, 3, and 4.

3.2. Real Samples Analysis. The validated method has been applied to analyze 67 cosmetics samples, including 18 gels, 25 ointments, and 24 creams taken from the oily skin and seborrhea treatment courts of make-up shops in our territory. All samples were processed according to the method described. The samples were analyzed and found as not containing any of the monitored steroids.
TABLE 3: Validation data of recovery for samples containing spiked standard solutions in blank cosmetic preparations.

\begin{tabular}{|c|c|c|}
\hline Steroids & Spiked conc. $\left(\mathrm{mg} \mathrm{kg}^{-1}\right)$ & $\%$ recovery \\
\hline \multirow{3}{*}{ CLP } & 0.5 & 98.9 \\
\hline & 1.0 & 99.0 \\
\hline & 1.5 & 99.1 \\
\hline \multirow{3}{*}{ DEX } & 0.5 & 94.3 \\
\hline & 1.0 & 96.2 \\
\hline & 1.5 & 96.8 \\
\hline \multirow{3}{*}{ MPD } & 0.5 & 98.8 \\
\hline & 1.0 & 97.6 \\
\hline & 1.5 & 99.1 \\
\hline \multirow{3}{*}{ PDL } & 0.5 & 95.5 \\
\hline & 1.0 & 96.3 \\
\hline & 1.5 & 97.7 \\
\hline \multirow{3}{*}{ FLA } & 0.5 & 92.1 \\
\hline & 1.0 & 93.4 \\
\hline & 1.5 & 92.9 \\
\hline \multirow{3}{*}{ FLM } & 0.5 & 97.6 \\
\hline & 1.0 & 99.1 \\
\hline & 1.5 & 98.8 \\
\hline \multirow{3}{*}{ PDN } & 0.5 & 99.2 \\
\hline & 1.0 & 99.5 \\
\hline & 1.5 & 99.0 \\
\hline \multirow{3}{*}{ TRM } & 0.5 & 93.1 \\
\hline & 1.0 & 92.6 \\
\hline & 1.5 & 94.2 \\
\hline \multirow{3}{*}{ TRA } & 0.5 & 92.4 \\
\hline & 1.0 & 91.9 \\
\hline & 1.5 & 93.0 \\
\hline \multirow{3}{*}{ BCL } & 0.5 & 96.4 \\
\hline & 1.0 & 93.4 \\
\hline & 1.5 & 94.7 \\
\hline
\end{tabular}

\section{Conclusions}

In this work a LC-MS/MS method was validated. The method is accurate, precise, and suitable for the determination of ten different active substances of the glucocorticoids family in counterfeit cosmetic products. The extractive process has been proven to be rapid, efficient, and suitable for preparations such as creams and ointments. The chromatographic method allowed an optimal separation of the analytes; furthermore the MS/MS detection ensured a univocal identification and an excellent sensitivity. The proposed method that mainly aimed at the accurate and reproducible determination of ten steroids was found to be useful for the quality control of pharmaceutical formulations and the screening of counterfeit cosmetic products suspected to contain steroids, which are banned in cosmetics. 
TABLE 4: Validation data of precision and accuracy for samples containing spiked standard solutions in blank cosmetic preparations. ${ }^{a} \mathrm{RSD}$ $(\%)=\left(\mathrm{SD} /\right.$ mean $\left.C_{\mathrm{obs}}\right) \times 100 .{ }^{\mathrm{b}}$ Accuracy $($ Bias $\%)=\left[\left(C_{\mathrm{obs}}-C_{\text {nom }}\right) / C_{\text {nom }}\right] \times 100$.

\begin{tabular}{|c|c|c|c|c|c|c|c|c|c|c|c|c|}
\hline \multirow{3}{*}{ Steroids } & \multicolumn{6}{|c|}{ Intraday analysis $(n=6)$} & \multicolumn{6}{|c|}{ Interday analysis $(n=18)$} \\
\hline & \multicolumn{3}{|c|}{ Precision $^{\mathrm{a}}$} & \multicolumn{3}{|c|}{ Accuracy $^{\mathrm{b}}$} & \multicolumn{3}{|c|}{ Precision $^{\mathrm{a}}$} & \multicolumn{3}{|c|}{ Accuracy $^{\mathrm{b}}$} \\
\hline & 0.5 & 1.0 & 1.5 & 0.5 & 1.0 & 1.5 & 0.5 & 1.0 & 1.5 & 0.5 & 1.0 & 1.5 \\
\hline CLP & 2.8 & 4.2 & 3.9 & 1.0 & 1.2 & 0.9 & 3.4 & 5.1 & 4.8 & 0.9 & 1.0 & 1.1 \\
\hline DEX & 3.1 & 4.1 & 6.1 & 2.8 & 2.4 & 2.1 & 4.2 & 5.3 & 6.9 & 1.9 & 1.8 & 2.4 \\
\hline MPD & 3.5 & 2.3 & 2.3 & 4.4 & 3.8 & 3.7 & 3.9 & 2.9 & 3.1 & 4.2 & 3.8 & 3.6 \\
\hline PDL & 2.6 & 3.4 & 2.4 & 1.2 & 1.4 & 1.2 & 2.1 & 3.9 & 2.9 & 1.8 & 1.5 & 1.1 \\
\hline FLA & 5.1 & 4.0 & 5.7 & 2.0 & 1.7 & 1.5 & 4.7 & 4.5 & 6.1 & 1.9 & 1.7 & 1.1 \\
\hline FLM & 4.0 & 3.5 & 2.6 & 2.7 & 1.8 & 1.9 & 5.2 & 4.7 & 4.5 & 1.9 & 1.8 & 1.9 \\
\hline PDN & 4.7 & 4.3 & 3.3 & 2.6 & 2.3 & 2.2 & 5.1 & 4.7 & 3.1 & 3.1 & 2.8 & 2.2 \\
\hline TRM & 2.9 & 3.9 & 2.6 & 3.4 & 2.8 & 2.2 & 3.3 & 4.2 & 3.8 & 3.8 & 3.8 & 4.2 \\
\hline TRA & 5.2 & 4.9 & 4.8 & 0.8 & 0.9 & 1.0 & 5.9 & 6.1 & 6.2 & 1.2 & 1.4 & 1.1 \\
\hline BCL & 6.1 & 5.9 & 5.6 & 6.0 & 5.4 & 5.2 & 6.5 & 6.3 & 6.8 & 4.5 & 6.1 & 5.0 \\
\hline
\end{tabular}

\section{Competing Interests}

The authors declare that they have no competing interests.

\section{References}

[1] J. D. Ference and A. R. Last, "Choosing topical corticosteroids," American Family Physician, vol. 79, no. 2, pp. 135-140, 2009.

[2] G. Committee and T. Force, "Guidelines of care for the use of topical glucocorticosteroids," Journal of the American Academy of Dermatology, vol. 35, no. 4, pp. 615-619, 2016.

[3] A. W. McKenzie, "Comparison of steroids by vasoconstriction," British Journal of Dermatology, vol. 78, no. 3, pp. 182-183, 1966.

[4] Facts and Comparisons, http://www.factsandcomparisons.com.

[5] A. C. Geraci, J. S. Crane, and B. A. Cunha, "Topical steroids; dosing forms and general considerations," Hospital Pharmacy, vol. 26, no. 8, pp. 699-719, 1991.

[6] B. G. Katzung, S. B. Masters, and A. J. Trevor, Basic \& Clinical Pharmacology. A Lange Medical Book, 2012.

[7] http://ec.europa.eu/growth/tools-databases/cosing/.

[8] S. Schoepe, H. Schäcke, E. May, and K. Asadullah, "Glucocorticoid therapy-induced skin atrophy," Experimental Dermatology, vol. 15, no. 6, pp. 406-420, 2006.

[9] N. N. Zöller, S. Kippenberger, D. Thaçi et al., "Evaluation of beneficial and adverse effects of glucocorticoids on a newly developed full-thickness skin model," Toxicology in Vitro, vol. 22, no. 3, pp. 747-759, 2008.

[10] S. Dyderski, E. Grześkowiak, E. Szalek, and A. Mrzyglód, "Pharmaceutical availability of clobetasol-17-propionate from cream and ointment," Acta Poloniae Pharmaceutica, vol. 58, no. 6, pp. 435-438, 2001.

[11] S. AbuRuz, J. Millership, L. Heaney, and J. McElnay, "Simple liquid chromatography method for the rapid simultaneous determination of prednisolone and cortisol in plasma and urine using hydrophilic lipophilic balanced solid phase extraction cartridges," Journal of Chromatography B: Analytical Technologies in the Biomedical and Life Sciences, vol. 798, no. 2, pp. 193201, 2003.

[12] V. A. Frerichs and K. M. Tornatore, "Determination of the glucocorticoids prednisone, prednisolone, dexamethasone, and cortisol in human serum using liquid chromatography coupled to tandem mass spectrometry," Journal of Chromatography B, vol. 802, no. 2, pp. 329-338, 2004.

[13] N. K. Hopkins, C. M. Wagner, J. Brisson, and T. E. Addison, "Validation of the simultaneous determination of methylprednisolone and methylprednisolone acetate in human plasma by high-performance liquid chromatography," Journal of Chromatography B: Biomedical Sciences and Applications, vol. 577, no. 1, pp. 87-93, 1992.

[14] Y. S. Nam, I. K. Kwon, and K.-B. Lee, "Monitoring of clobetasol propionate and betamethasone dipropionate as undeclared steroids in cosmetic products manufactured in Korea," Forensic Science International, vol. 210, no. 1-3, pp. 144-148, 2011.

[15] J. B. Golubovic, B. M. Otaševic, A. D. Protic, A. M. Stankovic, and M. L. Zečevic, "Liquid chromatography/tandem mass spectrometry for simultaneous determination of undeclared corticosteroids in cosmetic creams," Rapid Communications in Mass Spectrometry, vol. 29, no. 24, pp. 2319-2327, 2015.

[16] Y. S. Nam, I. K. Kwon, Y. Lee, and K.-B. Lee, "Quantitative monitoring of corticosteroids in cosmetic products manufactured in Korea using LC-MS/MS," Forensic Science International, vol. 220, no. 1-3, pp. e23-e28, 2012.

[17] W. Funk, V. Dammann, and G. Donnevert, Quality Assurance in Analytical Chemistry, 2007.

[18] http://www.uam.es/personal_pas/txrf/MU5.pdf.

[19] http://www.fda.gov/downloads/Drugs/GuidanceComplianceRegulatoryInformation/Guidances/ucm070107.pdf. 

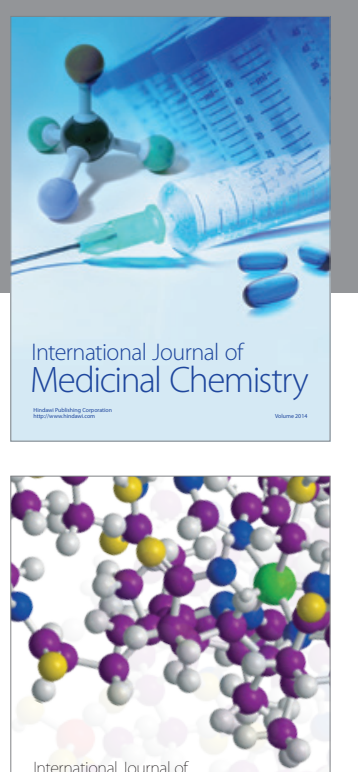

Carbohydrate Chemistry

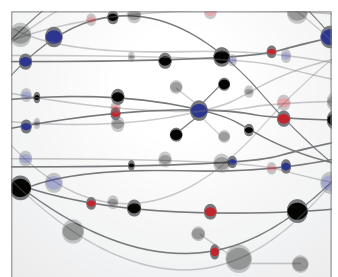

The Scientific World Journal
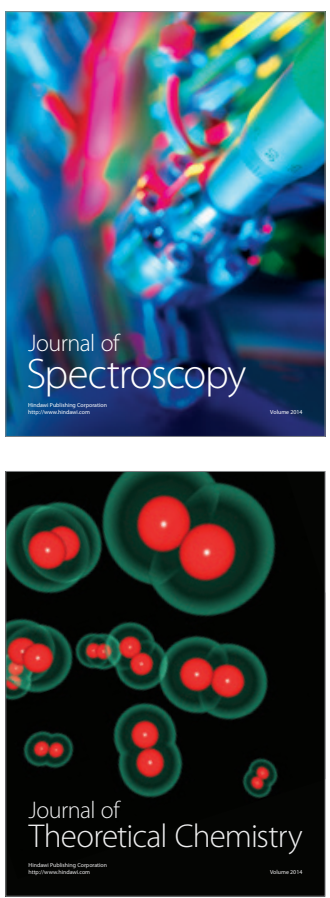
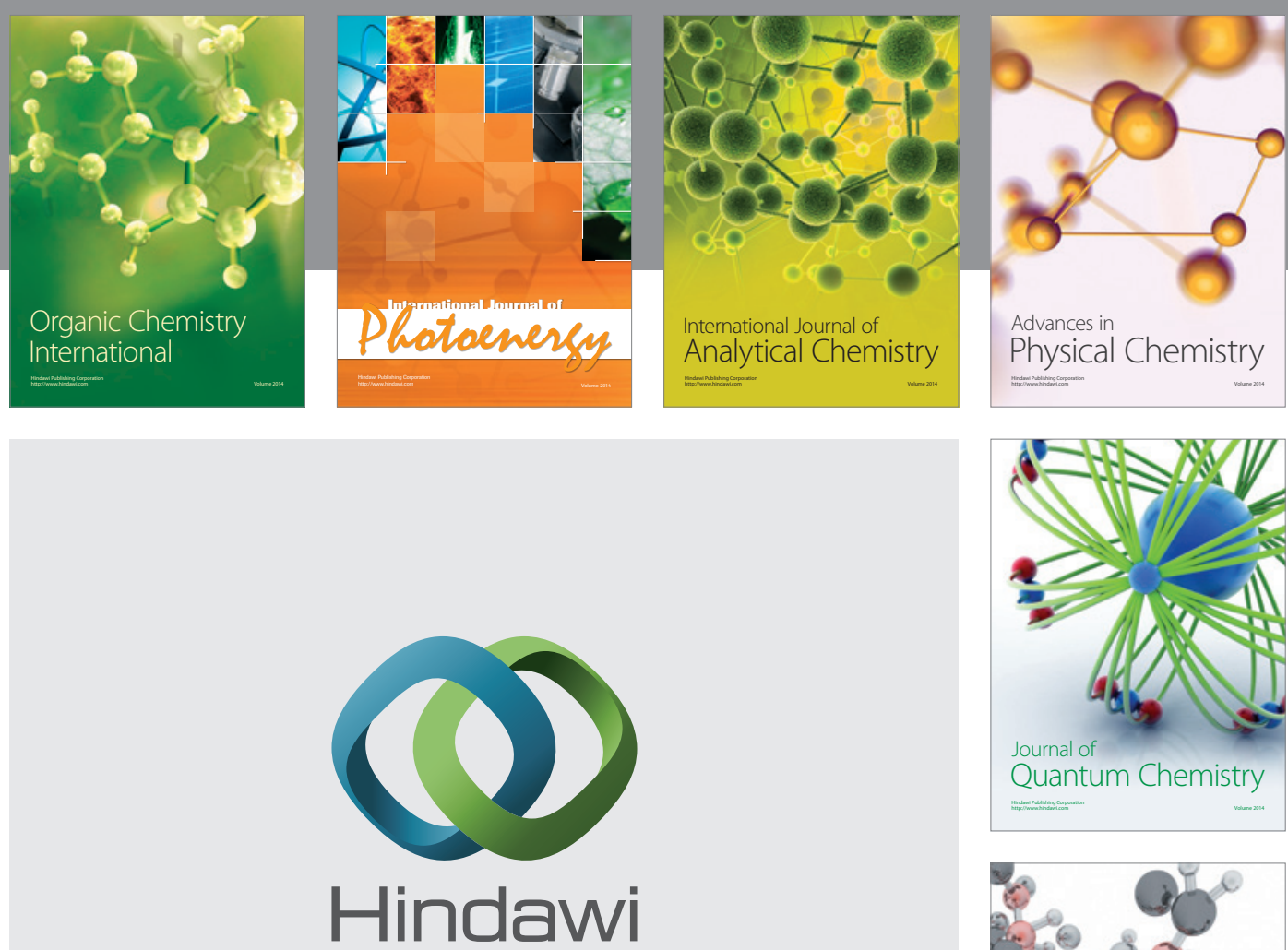

Submit your manuscripts at

https://www.hindawi.com

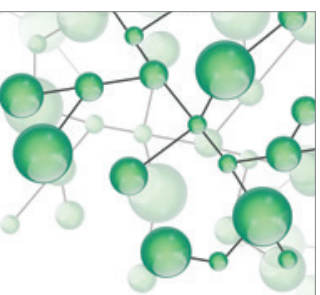

International Journal of

Inorganic Chemistry
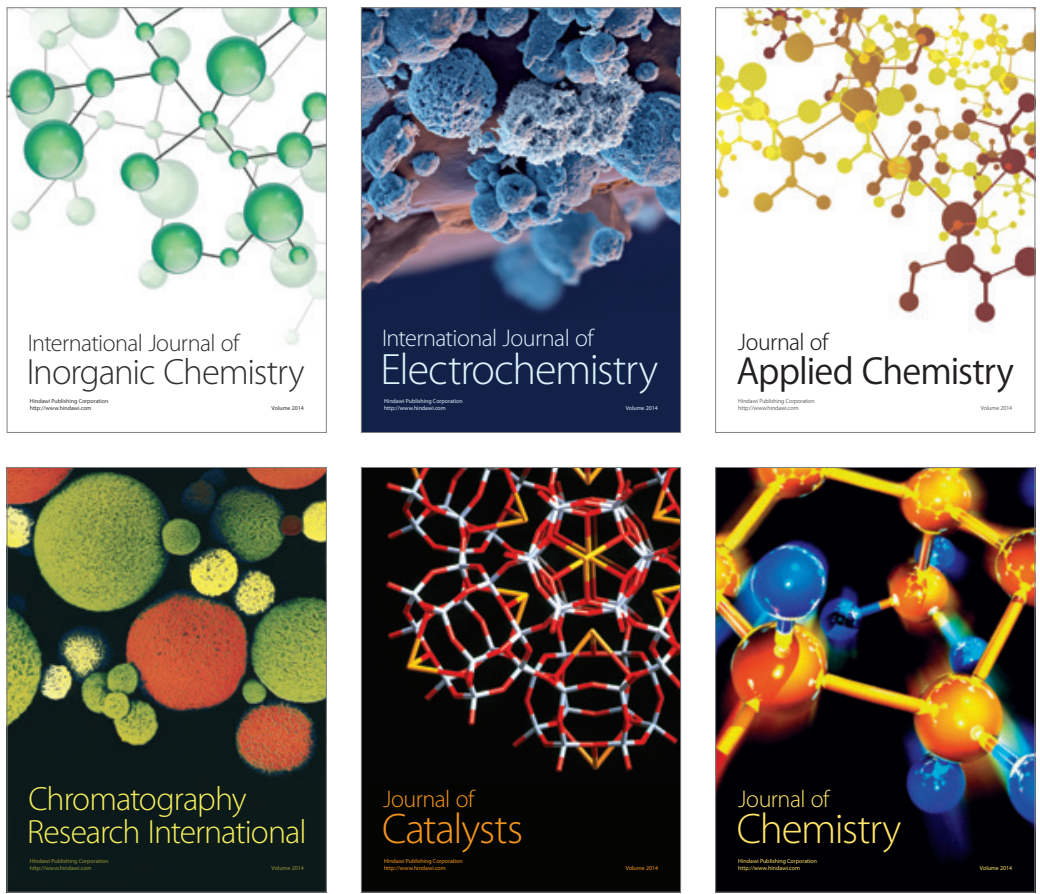

Journal of

Applied Chemistry
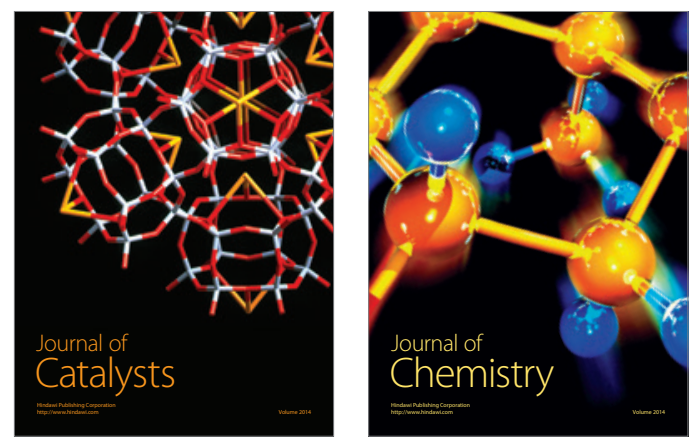
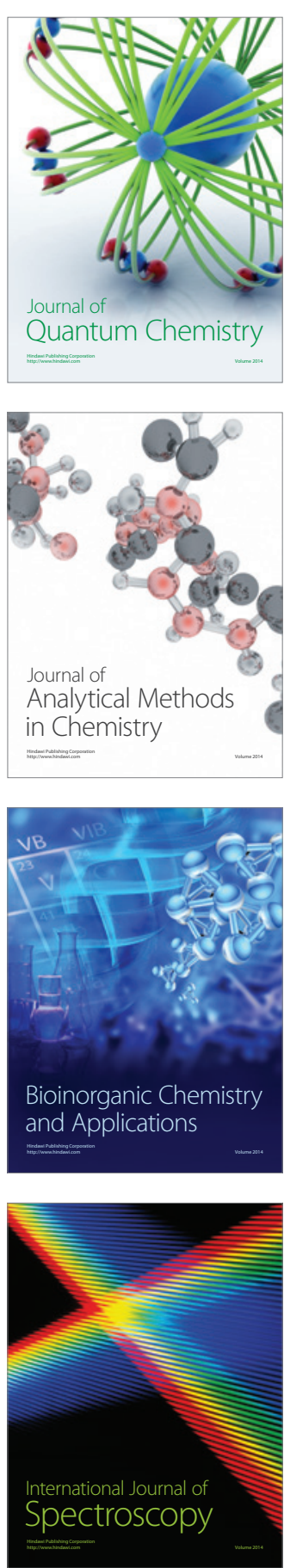